\title{
RESEARCH ARTICLE \\ INFLUENCE OF TIME-DOMAIN DETECTION ALGORITHM-BASED MOTION BIOLOGICAL IMAGE ANALYSIS ON SELECTING SUITABLE RECEIVING AREA
}

\author{
Carolina Viciana, Paul Jones \\ Department of Physical Education and Sport, Faculty of Sport Sciences, University of Patras, Greece. \\ *Corresponding Author Email:
}

This is an open access article distributed under the Creative Commons Attribution License, which permits unrestricted use, distribution, and reproduction in any medium, provided the original work is properly cited

\section{ARTICLE DETAILS}

\section{Article History:}

Received 16 November 2019 Accepted 20 Decmber 2019 Available online 8 January 2020

\section{ABSTRACT}

To comprehensively understand the variations in the joint angles and center of gravity under different suitable receiving situations of badminton. By constructing a time-domain detection algorithm, the variations in the joint angles and gravity center of joints in three different receiving areas are analyzed: (1) the right shoulder, elbow, and right wrist joint angles of forehand receiving in front of the net; (2) the right wrist and right shoulder joint flexionextension angels and the right elbow joint angle of backhand receiving in front of the net; and (3) the variations in center of gravity during high-shuttle receiving in front of the net. By quantifying the joint angles, the rationality of the selected area is determined. The results show that the proposed time-domain detection algorithm is accurate in judging the joint angles of the shoulder, elbow, and wrist under different receiving situations. As for the variations in joint angles of both forehand and backhand receiving in front of the net and the center of gravity during high-shuttle receiving, the judgments are close to the standard values. Also, the algorithm shows excellent stability while selecting the receiving areas. Based on the time-domain detection algorithm, the angle variations in the shoulder, elbow, and wrist joints in three different receiving areas are judged and selected, which provides suggestions for badminton training. The proposed algorithm is highly accurate and stable in selecting receiving areas in different situations. Also, selecting the shoulder, elbow, and wrist joint angles during exercise requires comprehensive consideration of multiple factors. A single parameter cannot make an accurate selection. The results have greatly improved the recognition of time-domain detection algorithm and joint angle variations while receiving the shuttle.

\section{KEYWORDS}

time-domain detection, badminton, receive, joint, angle

\section{INTRODUCTION}

With the gradual enrichment of materials and the improvement of living standards, people are becoming enthusiastic about spiritual entertainment and sports. Especially, the number of people who are interested in badminton is increasing [1]. The main reason is that badminton not only exercises the body but also promotes mutual exchanges, mutual feelings, and mutual understandings between the players [2]. Therefore, the masses of badminton in China are extremely wide. A critical factor in promoting badminton is that in China, the competitive level of badminton is high, which has achieved excellent results in international competitions. Therefore, the public is extremely enthusiastic about badminton. Also, badminton has relatively low requirements for the training courses, which makes it suitable for wide promotion [3].

However, in China, the places or courses for badminton are rare, and most of the amateur players have not mastered the correct practicing skills and standardized motions [4]. Such a situation has seriously hindered the healthy and sustainable development of badminton in China, as well as the stable development and cultivation of badminton talents. The main actions of badminton are receiving and hitting. If badminton players do not master these actions correctly, they cannot improve their competitive levels in badminton, which is a serious problem encountered in learning badminton [5]. If this problem cannot be effectively solved, the practicing efficiency of badminton players will be low, as well as the progress in badminton skills. Therefore, it should be valued by athletes and related departments [6].

Therefore, this study analyzes the biological images of badminton actions based on the time-domain detection algorithm, providing substantial help for the practice of badminton. The major contribution of this study is that by using a modified algorithm, the problems of finding proper receiving and hitting areas and the variations in joint angles of badminton players are solved, thereby improving the training methods of badminton in terms of shuttle receiving. The study of badminton is critical for the development of national fitness. In this way, the physical fitness of the public can be improved correctly and effectively [7].

In summary, this study explores the time-domain detection algorithm and biological images of badminton receiving motions. Also, this study investigates the angle variations in the joint angles in different receiving areas of badminton through the proposed algorithm. The results show that the time-domain detection algorithm has outstanding accuracy and stability in analyzing the joint angles of the receiving motions in badminton. The innovation of this study is applying the time-domain detection algorithm to analyze the biological images of badminton receiving actions and joint angle variations of players, which is significant for the in-depth exploration of the time-domain detection algorithm and badminton. The results of this study have provided a reference for future research. Therefore, this study is a valuable research topic. 


\section{METHODOLOGY}

\section{$2.1 \quad$ Research methods}

This study uses a combination of algorithm models and reference data to study the parameters of upper limb joints, torso, and lower limb joints during the receiving actions of badminton, as well as motion indexes such as movement displacements. Therefore, the standard requirements for the technical actions of receiving are obtained [8].

\subsection{Construction of time-domain algorithm}

The time-domain detection algorithm divides the current frame into a certain number of non-overlapping blocks, uses the previous frame as a reference frame, and finds the reference block closest to the current block in the reference frame according to certain principles and search strategies. The calculation equation is defined as:

$\mathrm{SAD}=\sum_{\mathrm{i}=0}^{\mathrm{I}} \sum_{\mathrm{j}=0}^{\mathrm{J}}\left|\mathrm{c}_{\mathrm{ij}}-\mathrm{p}_{\mathrm{ij}}\right|$

Where: $c_{i j}$ is the gray value of each pixel in the current macroblock, $p_{i j}$ is the gray value of each pixel in the reference macroblock. For objects with different motion intensities, the time-domain filter needs to use different filtering intensities, which can avoid the tailing phenomenon of fastmoving objects effectively. The motion intensity detection operator $S$ is defined as follows:

$\mathrm{S}=\frac{1}{\mathrm{n}}\left|\sum_{\mathrm{i}=0}^{\mathrm{I}} \sum_{\mathrm{j}=0}^{\mathrm{J}} \mathrm{c}_{\mathrm{ij}}-\sum_{\mathrm{i}=0}^{\mathrm{I}} \sum_{\mathrm{j}=0}^{\mathrm{J}} \mathrm{p}_{\mathrm{ij}}\right|$

It is assumed that the highest and lowest thresholds of macroblock motion intensity are $S_{h}$ and $S_{1}$. The motion intensity $S$ of each macroblock is defined in three situations:

(1) $S=S_{h}$, the macroblock is a violent motion with high motion intensity. The time-domain filtering intensity needs to be lowered, and the macroblock information is kept. (2) $\mathrm{S}_{\mathrm{l}}<\mathrm{S}<\mathrm{S}_{\mathrm{h}}$, the motion intensity of macroblock is in the middle state, and the filtering intensity needs to be moderate. (3) $S \leq S$, the macroblock is gentle motion with low motion intensity. The time-domain filtering intensity can be adjusted to remove the noise effectively.

In this study, the time-domain filter uses a first-order recursive filter structure, which continuously adds the images of the current frame and the reference frame with a certain weight ratio and performs multi-frame averaging, thereby reducing the impacts of noise. The recursive equation is as follows:

$\mathrm{F}_{\text {out }}(\mathrm{x})=\mathrm{k} \times \mathrm{F}_{\text {in }}(\mathrm{x})+(1-\mathrm{k}) \times \mathrm{F}_{\text {ref }}(\mathrm{x})$

Where: $\mathrm{x}$ is the coordinate of the macroblock, $\mathrm{F}_{\text {in }}$ is the pixel value of each point in the current macroblock, $F_{\text {ref }}$ is the pixel value of each point in the reference macroblock, and $\mathrm{F}_{\text {out }}$ is the pixel value of each point in the output macroblock. The current frame to be denoised is divided into blocks of 8 pixels $\times 8$ pixels, and the filtering is performed in block units. The steps of the algorithm are as follows:

(1) The motion estimation technology is used to find the reference macroblock that best matches the current macroblock in the previous frame, thereby determining the motion trajectory of the current macroblock in the time domain;

(2) The motion intensity of the object is calculated further, and the timedomain filtering intensity weight is dynamically adjusted according to the object motion intensity;

(3) The time-domain filter is used to perform weighted average filtering, complete the weighted average filtering process along the motion trajectory, and output the filtered denoised video images. The flowchart of the time-domain detection algorithm is shown in Figure 1.

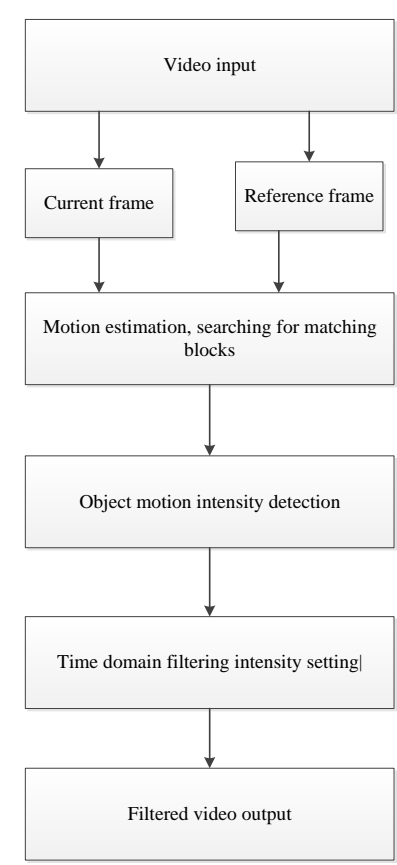

Figure 1: The workflow of the time-domain detection algorithm

\subsection{Application of time-domain detection algorithm in} badminton receiving

This study mainly uses the time-domain detection algorithm to study the displacements and angles of the shuttle-receiving joint in badminton. The angle features of the wrist joint: The sliced motion in front of the net is mainly powered by the wrist and fingers [9]. In the hitting stage, the amplitudes of the variations in the right wrist before and after the hitting in both players show that when the wrist is extended and retracted in the extended state, with the increase in flexion-extension amplitude, the wrist joint will do the forward flexion motion and the angle of the wrist joint will increase. Meanwhile, the flexion-extension angle of the wrist joint remains unchanged to reduce the upward force of the shuttle and control the height of the returning shuttle [10].

The angle features of the elbow joint: In the hitting stage, the elbow angles of both players are constantly increasing, with the amplitudes of flexion and extension respectively of $15^{\circ}$ and $9^{\circ}$, i.e., the arm stretches forward when hitting the badminton shuttle. The angle features of right shoulder: As for the flexion-extension angle of the right shoulder joint angel, when the arm is stretching forward, the should joint angle increases correspondingly. Meanwhile, the flexion angle of the right shoulder joint remains unchanged. Therefore, when hitting the badminton shuttle, players are powered with the forward-extension of the arm, the wrist, and the fingers. The speed features of gravity center: The speed of the center of gravity movements is consistent with the speed of the body movements, which can be seen from the variations in the moving speed of the center of gravity and the heads of players.

(1) Knee joint displacement during forehand receiving in front of the net: After calculation by the algorithm, the displacement range in the initial selection step is $650 \pm 110 \mathrm{~mm}$, the displacement range of the support link while receiving the badminton shuttle is $890 \pm 100 \mathrm{~mm}$, the displacement range of the contact link is $870 \pm 120 \mathrm{~mm}$, and the displacement range of the hitting link is $200 \pm 30 \mathrm{~mm}$. The amplitudes of angle variations are as follows: the right-hand flexion-extension angle is $120^{\circ}-170^{\circ}$, the right shoulder flexion-extension angle is $30^{\circ}-70^{\circ}$, and the right elbow joint angle is $100^{\circ}-130^{\circ}$.

(2) Knee joint displacement during backhand receiving in front of the net: After calculation by the algorithm, the displacement range in the initial selection step is $680 \pm 110 \mathrm{~mm}$, the displacement range of the support link while receiving the badminton shuttle is $800 \pm 90 \mathrm{~mm}$, the displacement range of the contact link is $820 \pm 30 \mathrm{~mm}$, and the displacement range of the hitting link is $292 \pm 50 \mathrm{~mm}$. The amplitudes of angle variations are as follows: the right shoulder joint angle is $30^{\circ}-80^{\circ}$, the elbow joint angle is $100^{\circ}-150^{\circ}$, and the right wrist joint angle is $150^{\circ}-120^{\circ}$. 
(3) Knee joint displacement during high-shuttle receiving: After calculation by the algorithm, the displacement range at the beginning of the selection step is $620 \pm 120 \mathrm{~mm}$, the displacement range of the support link while receiving the badminton shuttle is $780 \pm 50 \mathrm{~mm}$, the displacement range of the contact link is $800 \pm 32 \mathrm{~mm}$, and the displacement range of the hitting link is $300 \pm 100 \mathrm{~mm}$.

\section{RESULTS AND DISCUSSION}

The angle features of upper limb joint motion during forehand receiving in front of the net are shown in Figure 2. The biological images during forehand receiving in front of the net are shown in Figure 3. As shown in Figure 2, based on the judgment of the algorithm proposed in this study, during forehand receiving in front of the net, with the increase in time, the motion angles of upper limb joints, including upper right wrist joint flexion-extension angle, right shoulder flexion-extension angle, and right elbow joint angle, show different variation tendencies. The right shoulder flexion-extension angle and right elbow joint angle increase with the time, while the right wrist joint flexion-extension angle decreases with the time. The variation ranges are: right wrist joint flexion-extension angle is $120^{\circ}-$ $170^{\circ}$, the right shoulder flexion-extension angle is $30^{\circ}-70^{\circ}$, and right elbow joint angle is $100^{\circ}-130^{\circ}$. As shown in Figure 3, the biological images during forehand receiving in front of the net are divided into 5 steps: the preparation gesture, the judgment and initialization, stepping and swinging, hitting, and returning back. The correct completion of these 5 steps can realize forehand receiving in front of the net.

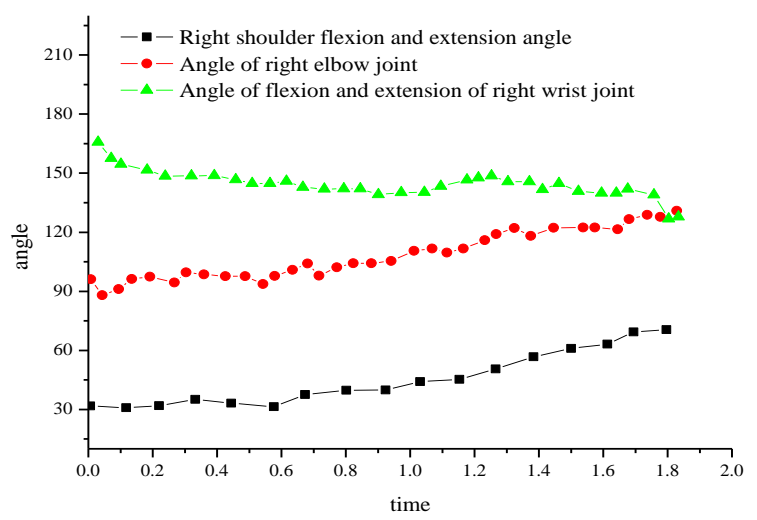

Figure 2: Angle features of upper limb motions of forehand receiving in front of the net

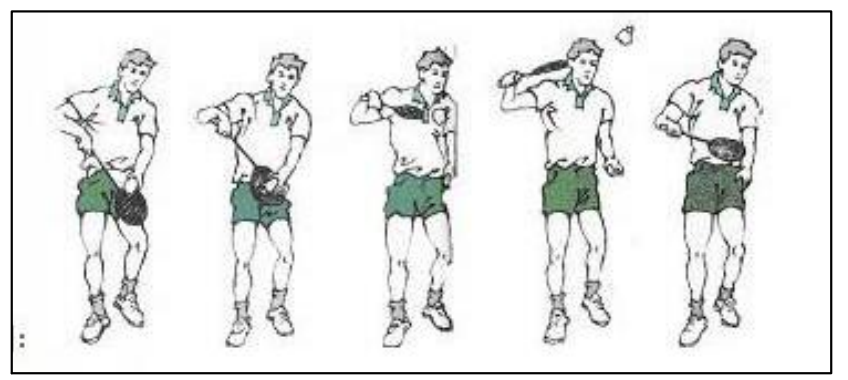

Figure 3: Biological images of forehand receiving in front of the net

The angle features of upper limb joint motion during backhand receiving in front of the net are shown in Figure 4. The biological images during backhand receiving in front of the net are shown in Figure 5. As shown in Figure 4, based on the judgment of the algorithm proposed in this study, during backhand receiving in front of the net, with the increase in time, the motion angles of upper limb joints, including right shoulder joint angle, elbow joint angle, and right wrist joint angle show different variation tendencies. The right shoulder angle and elbow joint angle increase with the time, while the right wrist joint angle decreases with the time. The variation ranges are: right shoulder joint angle is $30^{\circ}-80^{\circ}$, the elbow joint angle is $100^{\circ}-150^{\circ}$, and the right wrist joint angle is $150^{\circ}-120^{\circ}$. As shown in Figure 5, the biological images during backhand receiving in front of the net are divided into 4 steps: the judgment and initialization, stepping and swinging, hitting, and returning back. The correct completion of these 4 steps can realize backhand receiving in front of the net.

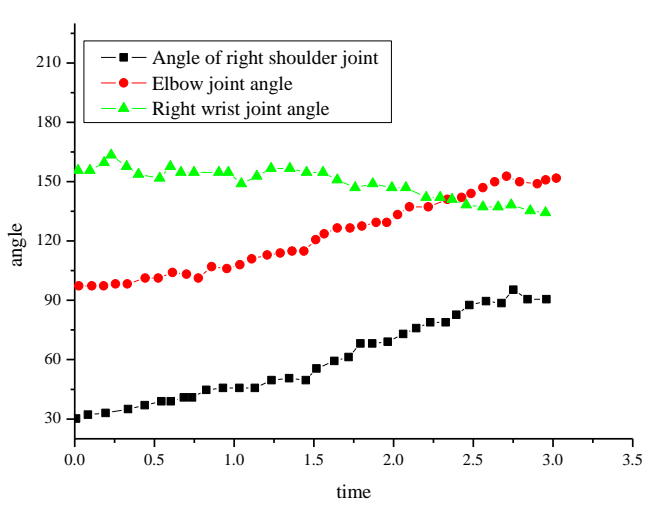

Figure 4: Angle features of upper limb motions of backhand receiving in front of the net

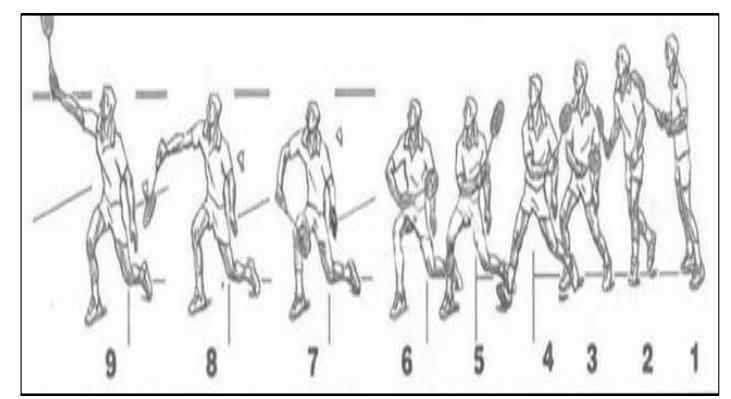

Figure 5: Biological images of backhand receiving in front of the net

The high-shuttle receiving variations in the center of gravity and the analysis of biological images of high-shuttle receiving are shown in Figure 6 and Figure 7, respectively. As shown in Figure 6, the research on badminton high-shuttle receiving variations in the center of gravity by time-domain detection algorithm shows that at the beginning, the body of players is first lowered. In the next stage, the body starts to rise, slightly lowers, and finally continues to rise. It has demonstrated that the timedomain detection algorithm is accurate and stable in analyzing the receiving areas and center of gravity in badminton. As shown in Figure 7 , the biological images during high-shuttle receiving are divided into 3 steps: stepping and swinging, hitting, and returning back. The correct completion of these 3 steps can realize backhand receiving in front of the net.

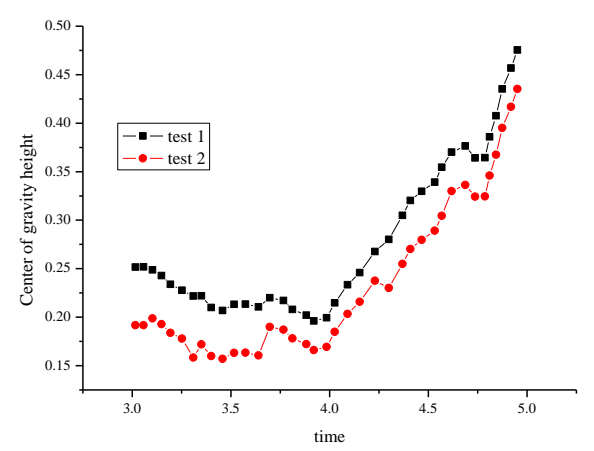

Figure 6: Variations in center of gravity during high-shuttle receiving

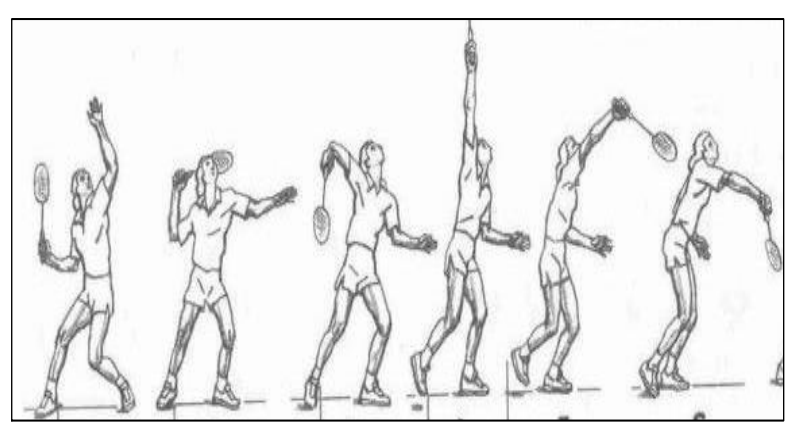

Figure 7: Biological images of high-shuttle receiving 


\section{CONCLUSION}

In this study, by using the time-domain detection algorithm, the biological images of badminton players during shuttle receiving are analyzed, the variations in joint angles of players under different receiving situations are investigated. The results show that for forehand receiving in front of the net, the flexion-extension angles of right wrist joint are $120^{\circ}-170^{\circ}$, the flexion-extension angles of the right shoulder are $30^{\circ}-70^{\circ}$, and the joint angles of the right elbow are $100^{\circ}-130^{\circ}$. For backhand receiving in front of the net, the joint angles of the right shoulder are $30^{\circ}-80^{\circ}$, the joint angles of the elbow are $100^{\circ}-150^{\circ}$, and the joint angles of right wrist are $150^{\circ}$ $120^{\circ}$. For high-shuttle receiving, the variations in the center of gravity are 0.4-0.5 m. Based on the time-domain detection algorithm, the research results of biological images show that the proposed algorithm has a prominent performance in analyzing the angle variations of multiple joints in various receiving areas of badminton.

Therefore, in this study, the time-domain detection algorithm is used to investigate the joint angle variations of badminton players under different receiving situations. The results show that the time-domain detection algorithm is accurate in determining the data of joint variations of the shuttle receiver. The proposed algorithm in this study can provide a valuable reference for badminton training. However, this study is subjected to certain limitations. For example, the analysis of the receiving area of badminton shuttles in actual situations is relatively complicated, involving multiple factors. While analyzing the algorithm, some factors are idealized to reduce the possible interferences. Therefore, the results obtained are slightly less convincing. The subsequent research should be more in-depth and detailed; therefore, the obtained results will be more comprehensive, which will reduce the interferences brought by other factors. The research in this study has essential reference value for future research.

\section{REFERENCES}

[1] Porter, E., Bahrami, H., Santorelli, A. 2016. A wearable microwave antenna array for time-domain breast tumor screening. IEEE transactions on medical imaging, 35(6), pp. 1501-1509.

[2] Litjens, G., Kooi, T., Bejnordi, B.E. 2017. A survey on deep learning in medical image analysis. Medical image analysis, 42, pp. 60-88.

[3] Rodrigues, D., Silva, G.F.A., Papa, J.P. 2016. EEG-based person identification through binary flower pollination algorithm. Expert Systems with Applications, 62, pp. 81-90.

[4] Havaei, M., Davy, A., Warde-Farley, D. 2017. Brain tumor segmentation with deep neural networks. Medical image analysis, 35, pp. 18-31.

[5] Albarqouni, S., Baur, C., Achilles, F. 2016. Aggnet: deep learning from crowds for mitosis detection in breast cancer histology images. IEEE transactions on medical imaging, 35(5), pp. 1313-1321.

[6] Graeser, M., Knopp, T., Szwargulski, P. 2017. Towards picogram detection of superparamagnetic iron-oxide particles using a gradiometric receive coil. Scientific reports, 7 (1), pp. 6872.

[7] Fierro, G.P.M., Meo, M. 2019. A combined linear and nonlinear ultrasound time-domain approach for impact damage detection in composite structures using a constructive nonlinear array technique. Ultrasonics, 93, pp. 43-62.

[8] Sunny, A.I., Tian, G.Y., Zhang, J. 2016. Low frequency (LF) RFID sensors and selective transient feature extraction for corrosion characterisation. Sensors and Actuators A: Physical, 241, pp. 34-43.

[9] von Diezmann, A., Shechtman, Y., Moerner, W.E. 2017. Threedimensional localization of single molecules for super-resolution imaging and single-particle tracking. Chemical reviews, 117 (11), pp. 7244-7275.

[10] Gonzalez-Valdes, B., Álvarez, Y., Rodriguez-Vaqueiro, Y. 2016. Millimeter wave imaging architecture for on-the-move whole body imaging. IEEE Transactions on Antennas and Propagation, 64(6), pp. 2328-2338. 